\title{
Application of radial electrical resistivity profile array for mapping deep vertical fractures in a proposed engineering construction site within the University of Ibadan Campus, Southwestern Nigeria
}

\author{
Olawale Olakunle Osinowo, Michael Oluseyi Falufosi \\ University of Ibadan, Department of Geology, Ibadan, Nigeria; \\ e-mail:wale.osinowo@ui.edu.ng, olawale.osinowo13@alumni.imperial.ac.uk (corresponding author)
}

(C) 2018 Authors. This is an open access publication, which can be used, distributed and reproduced in any medium according to the Creative Commons CC-BY 4.0 License requiring that the original work has been properly cited.

Received: 24 March 2018; accepted: 13 June 2018

\begin{abstract}
In this study we demonstrate the efficacy of radial electrical resistivity profile array in delineating deep narrow linear fracture that could constitute weakness in the subsurface, thus hindering foundation material in providing necessary support to proposed engineering construction, in the southeastern part of the University of Ibadan Campus, southwestern Nigeria, where nearby buildings show stress induced failure traceable to defect in foundation upon which the buildings were founded. Six electrical resistivity profile arrays were occupied at $0^{\circ} / 180^{\circ}, 30^{\circ} / 210^{\circ}, 60^{\circ} / 240^{\circ}, 90^{\circ} / 270,120^{\circ} / 300^{\circ}$ and $150^{\circ} / 330^{\circ}$ orientations to complete a clockwise $360^{\circ}$ sweep at $30^{\circ}$ interval. The radial profile array enabled subsurface features to be intersected at relatively high angle, thus making measurement of linear features of limited lateral extent feasible irrespective of their orientations. Low resistivity distribution which decreases in value with depth $(300-8 \Omega \mathrm{m})$ characterizes the northern part of the study area while relatively higher value that increases with depth $(420-4150 \Omega \mathrm{m})$ dominates the south. The occurrence of a narrow strip of low resistivity zone $(8-170 \Omega \mathrm{m})$ at $20 \mathrm{~m}$, which gains prominence with depth, dividing relatively resistive zone in the southern part of the study area indicate the presence of a deep vertical fracture whose detection is essential to guide further foundation geotechnical studies as well as aid the design of appropriate foundation that would support the proposed engineering structure.
\end{abstract}

Keywords: radial profile array, vertical fracture, narrow azimuth survey, proposed construction site

\section{INTRODUCTION}

The accuracy of geophysical technique to properly image the subsurface, especially to delineate salient features which oftentimes have limited lateral and vertical extent, depends largely on the sampling interval and acquisition geometry (Pazdirek \& Blaha 1996, Dahlin \& Zhou 2004, Stummer et al. 2004, Hennig et al. 2008). Deep vertical fractures (faults and joints) are difficult to image because of their limited lateral contrast that makes sampling across the structure more of a matter of chance in conventional parallel (narrow azimuth) geophysical profile array (Seaton \& Burbey 2002, Rønninget al. 2003). This is especially difficult where the fracture runs perpendicular to the general geological strike, since most profiles are usually established perpendicular to the general geologic strike and thus parallel to such linear feature. Fractures which often present contrasting lower 
resistivity values, especially when saturated with water are easy to delineate when the data acquisition geometry intersects the narrow linear feature through sampling at high angle. Radial geophysical profile array offers geophysical measurement pattern that enables all subsurface features to be intersected at relatively high angle (often as high as $45^{\circ}$ and above) which present near perpendicular intersection of the target subsurface features irrespective of their orientations. This is particularly important for ease of detecting narrow subsurface features which are sometimes limited in lateral extent. Thus it is very effective for mapping linear features such as fractures, veins and faults, some of which are significant to investigation objectives, be it, economic mineral exploration, hydrogeophysical investigation or engineering geophysical studies.

The electrical resistivity study of a proposed construction site in southeastern part of the University of Ibadan Campus, southwestern Nigeria was undertaken to delineate hidden structural feature(s) that could constitute subsurface zone of weakness with potential to affect the proposed engineering construction. This is very essential since adjacent structures such as buildings and walls present evidence of stress induced cracks and other associated failure features that could be related to defect in the foundation support offered by the subsurface rocks upon which they were founded (Fig. 1).

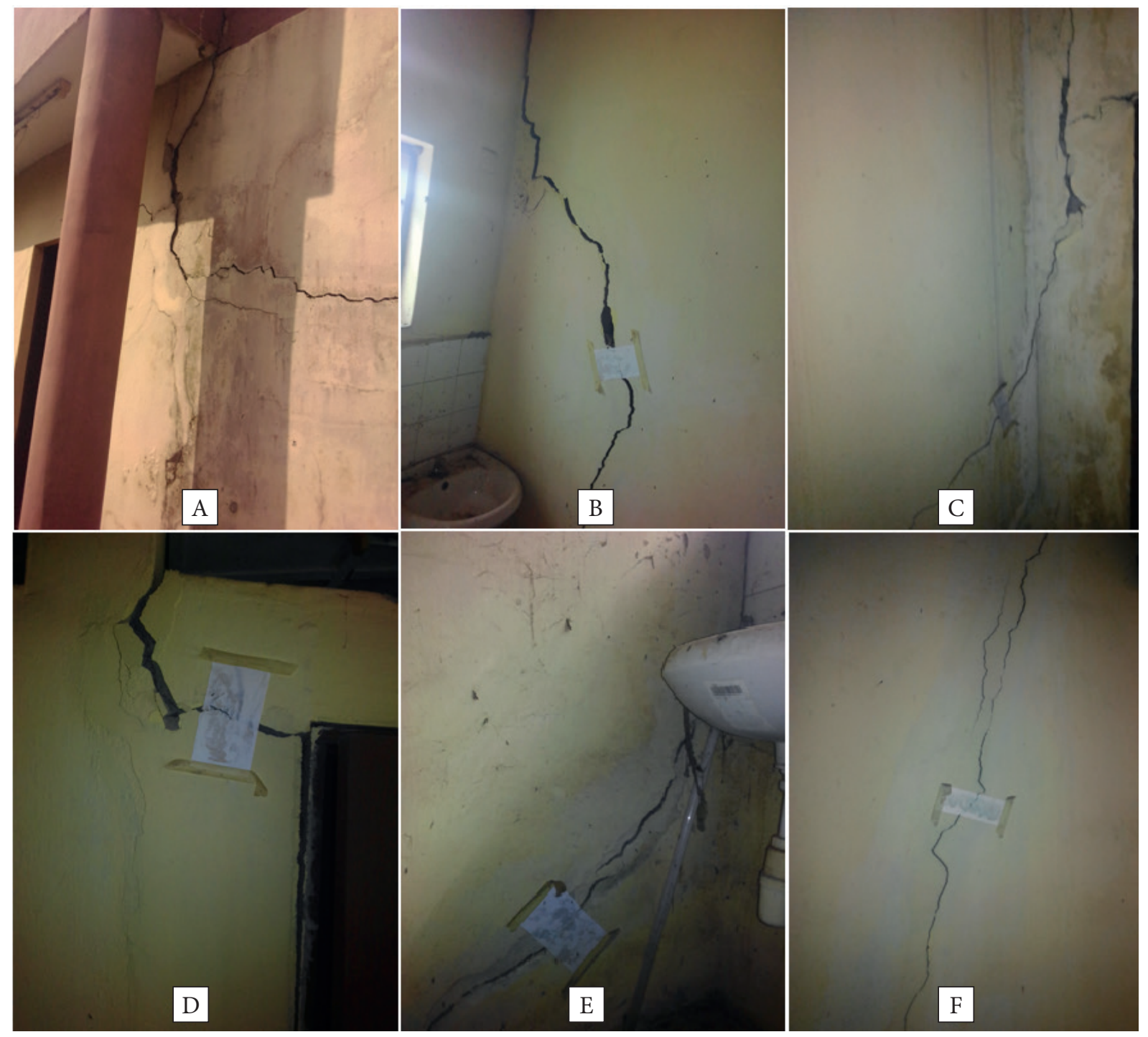

Fig. 1. Pictures of stress induced cracks in adjacent building located about $70 \mathrm{~m}$ southeast of the proposed construction site 


\section{GEOLOGY OF THE STUDY AREA}

The geologic terrain of Ibadan falls under the $\mathrm{Ni}$ gerian Basement Complex with migmatite-gneiss, quartzite, schist and granite being the dominate rock types (Elueze \& Okunlola 2003). The Nigerian Schist Belt (ca. 600 ma), which trends approximately North-South within the western half of the country, runs through Ibadan. The study area is located within the University of Ibadan Campus and the underlying rock types include quartzite and quartz-schist rocks underlain by banded gneiss rock unit which outcrop at some locations within the Campus. Schist and quartzite rock units sometimes share both lateral and vertical tectonic boundaries with banded-gneiss rocks and they all alligned in the north-south direction. The main lithologic units in the schistose rocks are quartzites and schists and they are associated with the migmatite-gneiss rock units. They alternate with each other and adjacent schistose-quartzite/migmatite-gneiss boundaries are somewhat parallel. Figure 2 presents the geologic map of the University of Ibadan Campus showing the study area.

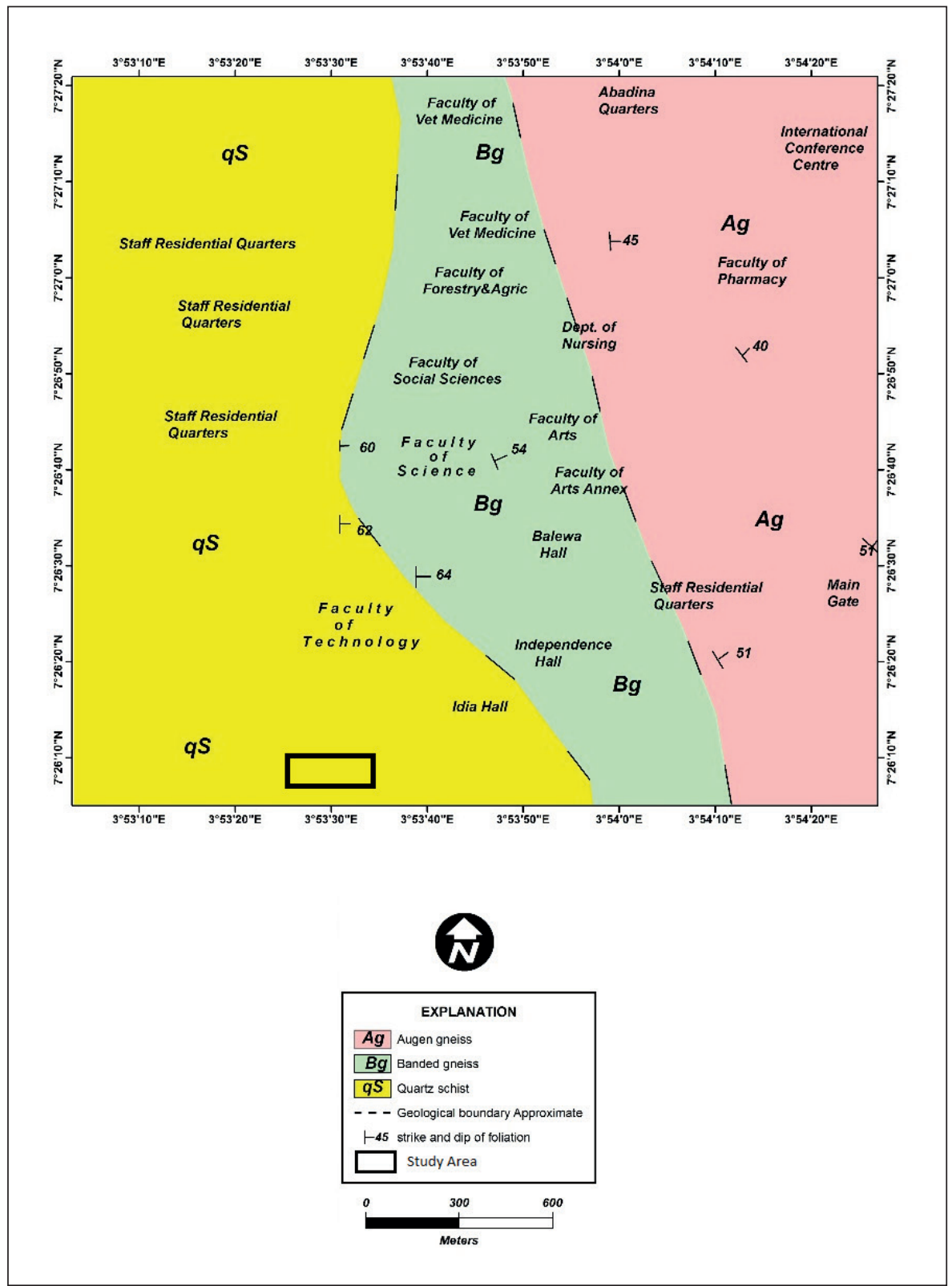

Fig. 2. Geologic map of the study area 


\section{MATERIAL AND METHODS}

The electrical resistivity survey was carried out with the SuperSting R8/IP/SP Geo-Electrical Tomography Instrument (Fig. 3). Ground resistivity measurement was done via four (4) electrodes, where current was injected into the subsurface by 12 volt battery powered resistivity meter, through two (2) current electrodes and the response measured with the aid of two (2) potential electrodes. The ratio of the injected current to the potential difference generated due to the flow of current in the subsurface indicates ground resistance to flow of electricity in the subsurface, which depends on many subsurface factors such as rock mineralogy, pore space or subsurface fractures and degree of saturation among others. The subsurface apparent resistivity is obtained when the geometric factor of the used electrode configuration is incorporated. In this study, dipole-dipole electrode configuration was adopted because of the combined advantage of high horizontal resolution and great depth of penetration. The high horizontal resolution is related to minimal or no inductive coupling effect which usually results when current and potential electrodes are established too close to each other (White \& Loke 2003). The radial profile array was adopted with the electrodes profile layout moved over a regular interval sweep of $30^{\circ}$ in a clockwise direction (Fig. 4). The SuperSting resistivity meter uses 8-channel multi-electrode automated system capable of automatic switch between individual electrodes to take resistivity measurement along 84 steel electrodes on the profile. A constant station separation interval of $1.5 \mathrm{~m}$ was maintained between the electrodes. A total of six profiles were occupied at $0^{\circ} / 180^{\circ}, 30^{\circ} / 210^{\circ}, 60^{\circ} / 240^{\circ}, 90^{\circ} / 270^{\circ}, 120^{\circ} / 300^{\circ}$ and $150^{\circ} / 330^{\circ}$ to complete a $360^{\circ}$ sweep at $30^{\circ}$ interval.
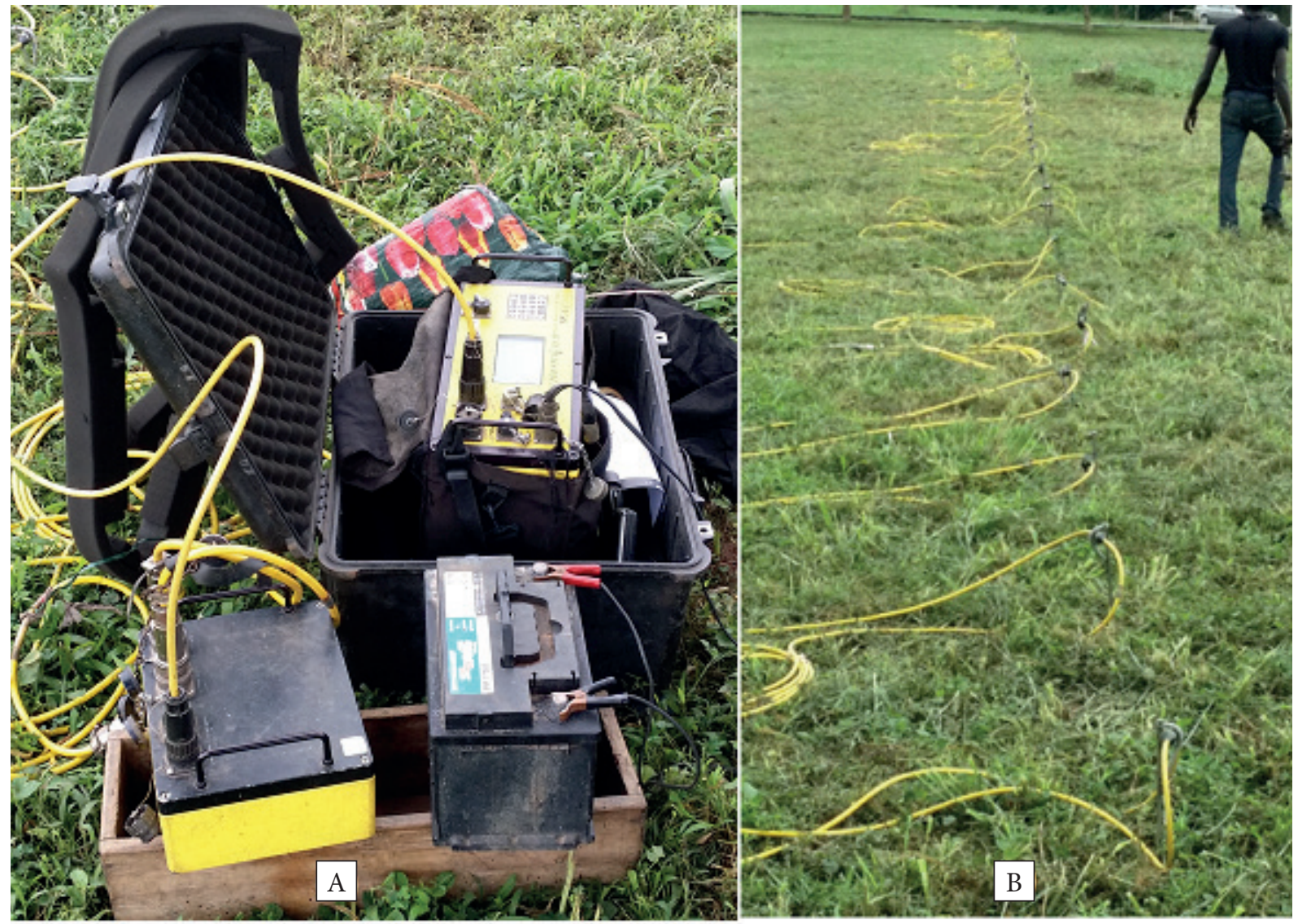

Fig. 3. Electrical resistivity meter (A) with AGI electrode switch box and 12 volt battery power source (B) one of the six radial electrical resistivity profile 


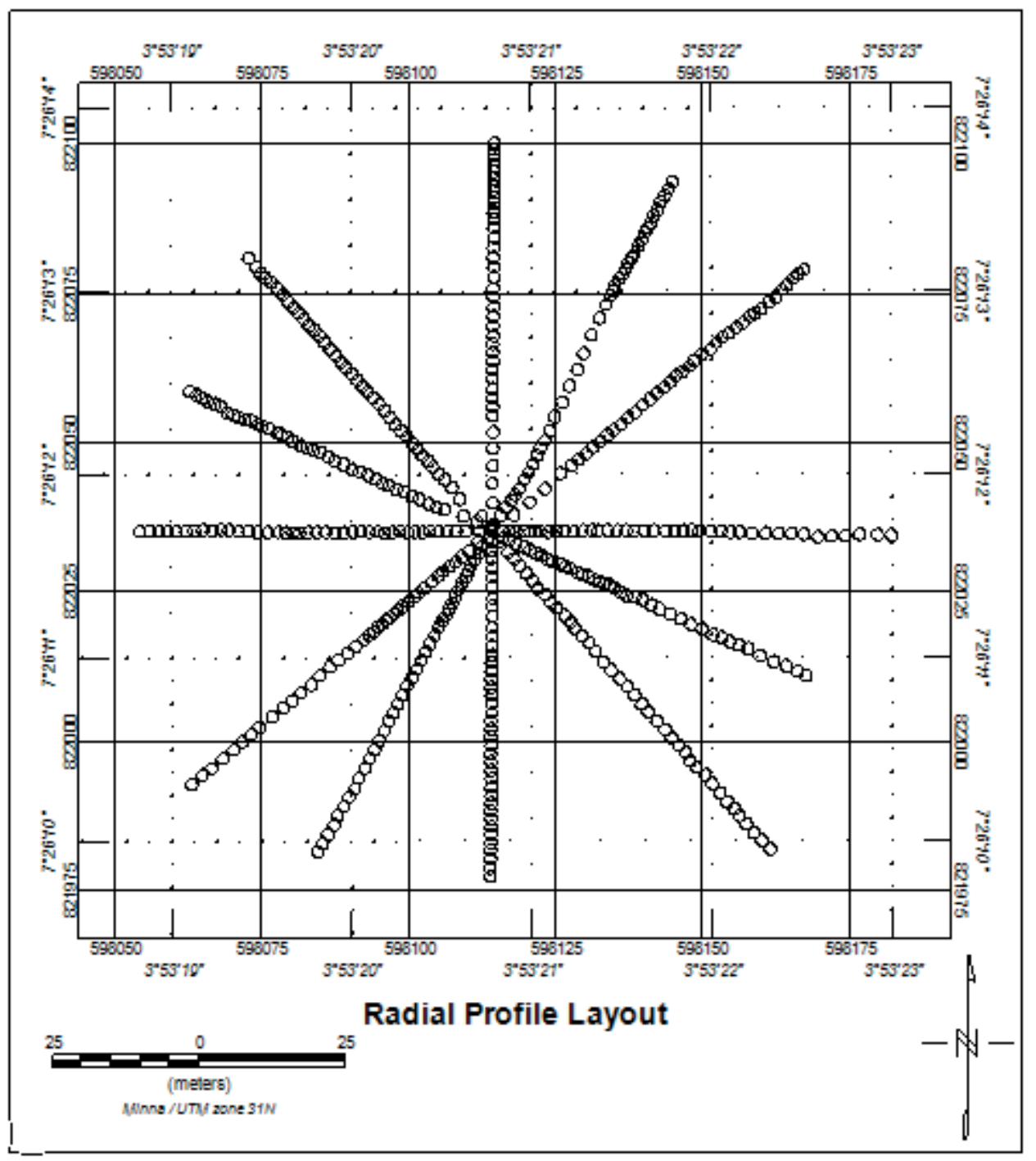

Fig. 4. Map showing the radial electrical resistivity profile layout

\section{DATA PROCESSING}

The measured apparent resistivity data obtained from individual profile were evaluated for errors (QC) and corrected for spurious readings and dead channels. Apparent resistivity data from individual profiles were inverted using RES2DINV inversion algorithms developed by Loke \& Baker (1996). The algorithm employs forward modeling subroutine to determine the apparent ground resistivity and thereafter uses a non-linear least squares optimisation techniques to invert the apparent ground resistivity data, clean the data by filtering out noisy data and effect correct depth matching based on electrode spacing to generate a two-dimensional (2D) true ground resistivity model of the subsurface (Sasaki 1989, Loke 1999, 2000, Loke et al. 2013). The resultant inverted resistivity data from individual profiles were depth sorted into their corresponding depths of measurements as indicated in the inverted resistivity data. The corresponding depth presented in the inverted data is the corrected depth matched data by the inversion algorithm which uses the half of the current electrode separation as the starting depth value but afterward reduced to less than $30 \%$ of the current electrode separation (Evjen 1938, Roy \& Apparao 1971, Inman et al. 1973, Banerjee \& Pal 1986). The depth sorted data were further georeferenced using the GPS acquired longitude and 
latitude values for each electrode station to define the location of the measured points. All data corresponding to different measurement depths properly georeferenced on each occupied profiles were combined and gridded using minimum curvature gridding technique which is ideal for line oriented data (Briggs 1974) to generate radial resistivity distribution across the study area.

\section{RESULTS AND INTERPRETATION}

The results of subsurface resistivity investigation which aimed at mapping deep linear structures are presented as radial 2D resistivity sections, sliced $3 \mathrm{D}$ subsurface resistivity model and iso resistivity surfaces as well as radial iso-depth resistivity distribution images of the study area. Figure 5 presents the $2 \mathrm{D}$ sections generated at different angles of $0^{\circ} / 180^{\circ}, 30^{\circ} / 210^{\circ}, 45^{\circ} / 225^{\circ}, 60^{\circ} / 240^{\circ}, 90^{\circ} / 270^{\circ}$, $120^{\circ} / 300^{\circ}, 135^{\circ} / 315^{\circ}$ and $150^{\circ} / 330^{\circ}$, with additional $2 \mathrm{D}$ resistivity distribution sections generated at $45^{\circ} / 225^{\circ}$ and $135^{\circ} / 315^{\circ}$ to observe possible variation at $15^{\circ}$ between $30^{\circ} / 210^{\circ}$ and $60^{\circ} / 240^{\circ}$ and $120^{\circ} / 300^{\circ}$ and $150^{\circ} / 330^{\circ}$, respectively. The sections were laid on the radial resistivity distribution image at $30 \mathrm{~m}$ depth for ease of comparison. The figure indicates low resistivity distribution in the north as opposed to higher values in the southern part of the study area, which suggests that almost all the profiles straddles the low resistive rock unit(s) in the north and highly resistive subsurface in the south. The sections also indicate that the resistivity distribution in the extreme north of the study area generally decreases with depth while the opposite, that is, increase in resistivity value with depth generally characterizes the south, except for narrow strip of low resistivity zone observed at relatively considerable depth $(>20 \mathrm{~m}$ depth). A careful observation of the sections and the iso-depth resistivity image generated at $30 \mathrm{~m}$ depth indicate that the deep narrow low resistivity zone delineated in the southern part of the study area is made possible because two of the occupied profiles run across the low resistivity zone, intersecting the fracture at relatively high angle. This is possible because the radial profile array is able to run fairly perpendicular to both the lithologic and structural features in the field, thus crosscutting small features despite its limited lateral spread, as in the case of the vertical fractures and faults.

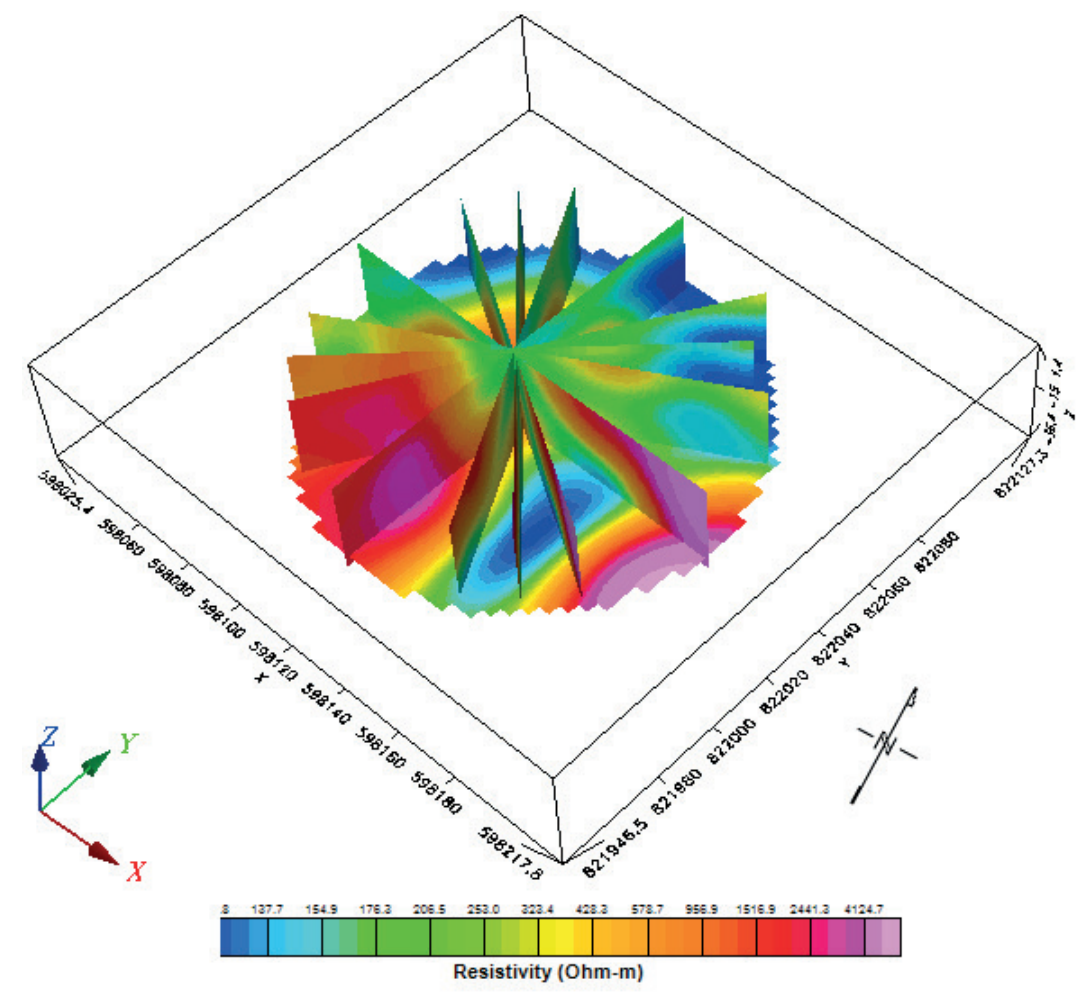

Fig. 5. 2D electrical resistivity sections acquired along radial profiles 
Sliced 3D radial subsurface resistivity models (sliced along SW - NE) and 3D iso-resistivity surface across the study area are presented in Figures 6. The figures show deep narrow but prominent low resistive zone, having limited lateral extent that decreases in resistivity (conductivity increase) value with depth, underlying the massive and relatively extensive zone of high resistivity. This suggests occurrence of deep vertical fault or fracture which does not extend to the surface. The vertical fault is further defined by the occurrence of narrow strip of conductive zone at depth dividing relatively extensive resistivity zone into two.

A

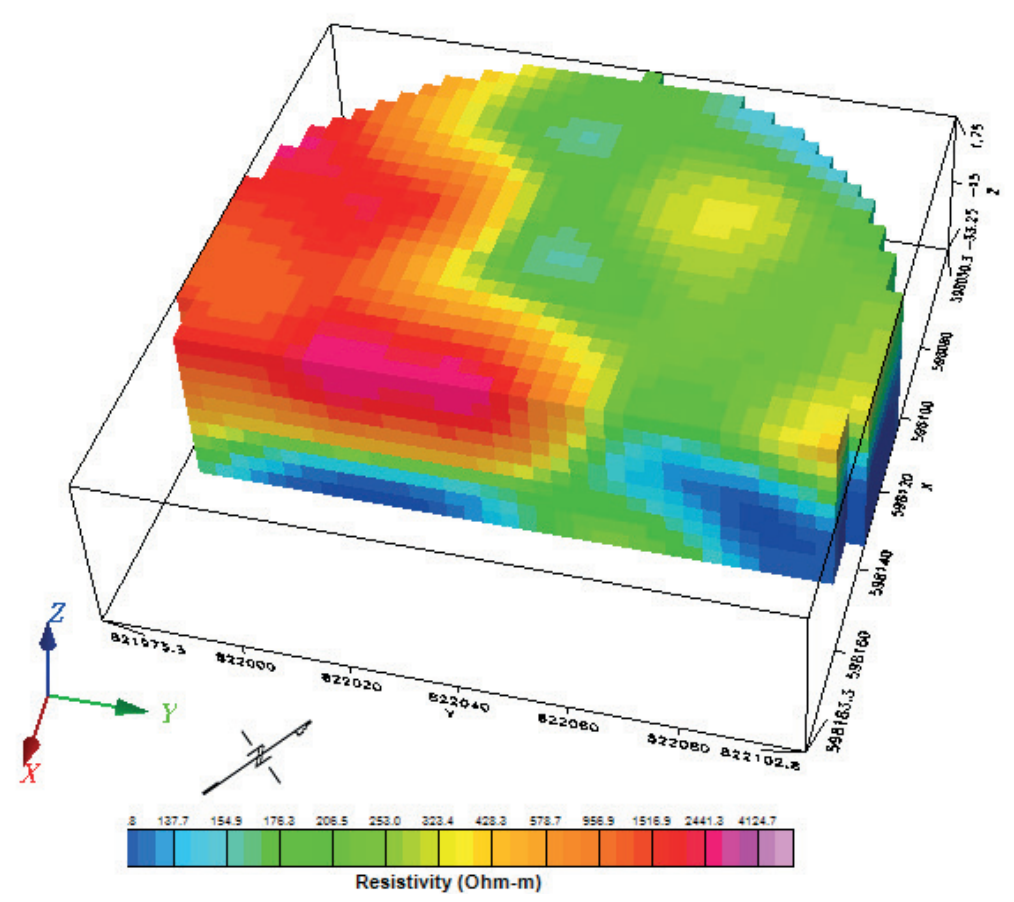

B

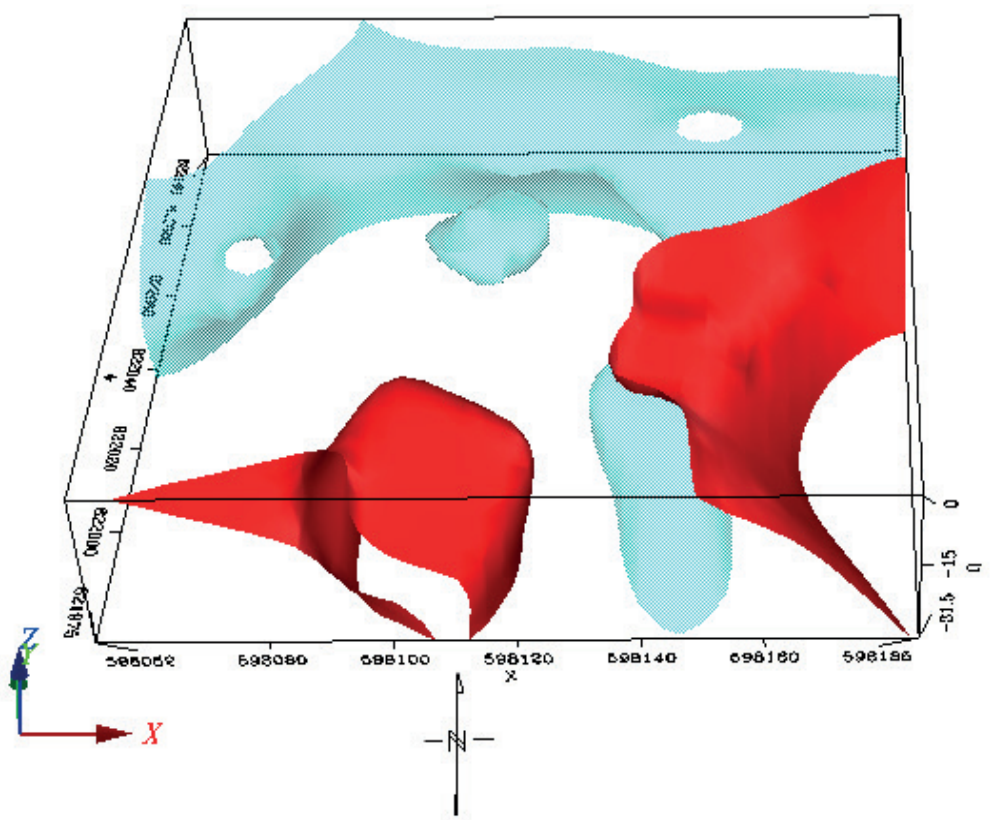

Fig. 6. Sliced 3D radial electrical resistivity model (sliced approximately $S W-N E)(A) ; 3 D$ iso-resistivity surface showing the narrow less resistive zone at depth underlying the resistive zone (B) 
Individual iso-depth radial subsurface resistivity distribution images obtained at $\sim 0 \mathrm{~m}$, at $5 \mathrm{~m}$, $10 \mathrm{~m}, 15 \mathrm{~m}, 20 \mathrm{~m}, 25 \mathrm{~m}$, and $30 \mathrm{~m}$ depths are presented in Figure 7A-G. The figures generally indicate regions of relatively low ground resistivity in the north of the study area while higher resistivity values characterize the south. The near surface and shallower part of the subsurface of the study area presents medium to relatively high resistivity distribution value ranging from $180 \Omega \mathrm{m}$ in the north to about $1000 \Omega \mathrm{m}$ in the south. However with increase in depth, some zones characterized with medium resistivity values in the north of the study area became less resistive (conductive) presenting lower resistivity values (130-8 $\Omega \mathrm{m}$ ). Likewise, some of the zones that show relatively high near surface resistivity distribution also present higher resistivity $(>2800 \Omega \mathrm{m})$ value at depth. The coverage of zone of relatively high resistivity, which appears restricted more to the southern part of the study area decreases with depth in the south, but gained prominence northwards as it spreads more northward, indicating resistivity increasing with depth in some part of the northern section of the study area.

The elongate lower resistivity zone $(\leq 45 \Omega \mathrm{m})$ becomes apparent in the south at $25 \mathrm{~m}$ and $30 \mathrm{~m}$ depth. The increase in the resistivity as well as the distribution/spread of the elongate zone with depth suggests that the structure will become more prominent with depth beyond $30 \mathrm{~m}$. The configuration of the elongate low resistivity zone suggests that it is likely to be a deep fault or fracture that cuts through the resistive crystalline rock, identified from local geology of the study area to be banded gneiss rock in the south that bounds the quartzite and quartz-schist rocks in the north.
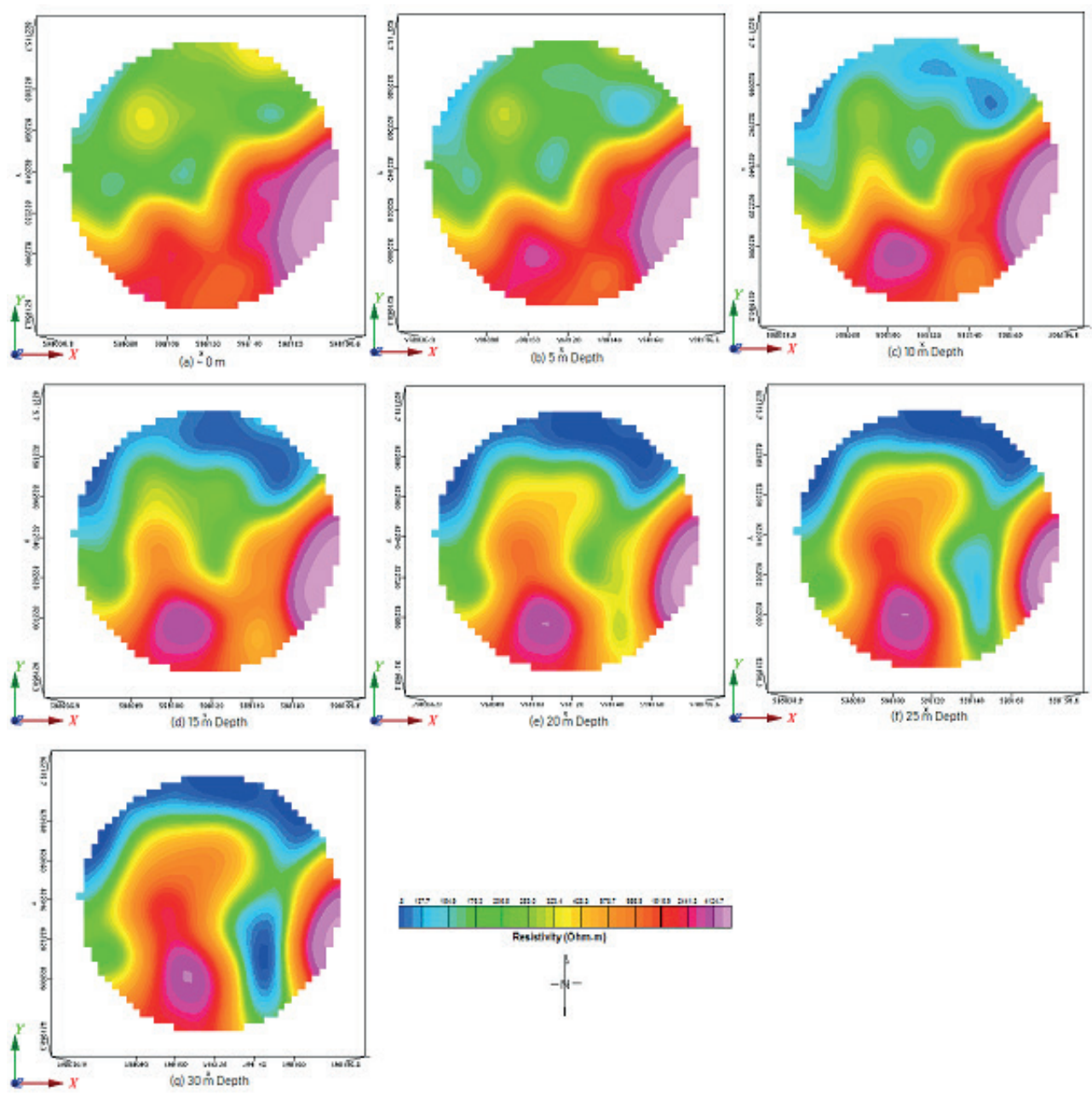

Fig. 7. Iso-depth radial electrical resistivity distributions at $\sim 0 \mathrm{~m}, 5 \mathrm{~m}, 10 \mathrm{~m}, 15 \mathrm{~m}, 20 \mathrm{~m}, 25 \mathrm{~m}$ and $30 \mathrm{~m}$ depths below the surface 
The subsurface resistivity distribution across the proposed construction site as shown by the generated subsurface sections and iso-depth radial resistivity images confirm relatively low resistivity value which decreases with depth in the northern part of the study area while higher resistivity distribution that generally increases with depth characterize the south. However, the relatively high resistivity distribution in the south is interjected at about $25 \mathrm{~m}$ depth by narrow zone of low resistivity value, which becomes decreasingly resistive (conductive) with depth (Figs. 5-7). This zone of low resistivity distribution at depth will likely constitute a zone of weakness that could hinder the subsurface giving necessary foundation support to the proposed building. This is because rock conductivity is mainly through galvanic conduction which depends to a large extent on rock mineralogy and degree of water saturation. Since crystalline rocks can only hold water within fractures, occurrence of low resistivity zone at depth, especially when it is overlain and flanked by resistive units as shown in the presented results (Figs. 6,7) suggest occurrence of water saturated fractures at depth. Water saturated fractures will constitute zone of weakness as the joints and faults within the rocks will introduce rock weakness through loss of rock cohesion across their surfaces. Further still, the water will aid relatively higher weathering of rocks in the precinct of the fractures than the un-fractured part of the subsurface.

\section{CONCLUSION}

The strength of radial profile array based on the radial acquisition geometry that intersects subsurface features in nearly perpendicular manner has been heighted in this study. The radial electrical resistivity acquisition profile array has proved efficient in delineating deep vertical fracture/ fault which based on its limited lateral extent and depth of occurrence would be difficult to image by conventional parallel (narrow azimuth) profile array. Identification of deep subsurface fractures is very essential for a proposed construction site where fractures/faults induced structural discontinuities and rock association have been identified to cause structural failures in surrounding existing buildings in the southeastern part of University of Ibadan Campus, southwestern Nigeria. The generated subsurface electrical resistivity distribution images, sections and model present a radial snap into the surface which offers vital information necessary for the design of appropriate foundation support for the proposed building.

The authors wish to express thanks to Messrs. Fogofoluwa Adeoye, and Seni Oladokun for helping out during the data acquisition phase of this study. The anonymous reviewers are highly appreciated for offering very useful suggestions and contributions that helped improved the quality of this article.

\section{REFERENCES}

Banerjee B. \& Pal B.A., 1986. A simple method for determination of depth of investigation characteristics in resistivity prospecting. Exploration Geophysics, 17, 93-95.

Briggs I.C., 1974. Machine contouring using minimum curvature. Geophysics, 39,39-48.

Dahlin T. \& Zhou B., 2004. A numerical comparison of 2D resistivity imaging with 10 electrode arrays. Geophysical Prospecting, 52, 5, 379-398.

Elueze A.A.\& Okunlola O.A., 2003. Petrochemical and Petrogenetic Characteristics of Metasedimentary Rocks of Lokoja-Jakura Schist Belt, Central Nigeria. Journal of Mining and Geology, 39, 1, 21-27.

Evjen H.M., 1938. Depth factor and resolving power of electrical measurements. Geophysics, 3, 78-85.

Hennig T., Weller A. \& Möller M., 2008. Object orientated focussing of geoelectrical multielectrode measurements. Journal of Applied Geophysics, 65, 2, 57-64.

Inman J.R., Ryu J. \& Ward S.H., 1973. Resistivity inversion. Geophysics, 38, 6, 1088-1108.

Loke M.H., 1999. A practical guide to $2 D$ and 3D surveys. Electrical imaging surveys for environmental and engineering studies. Geotomo software, Malaysia, 8-10, [on-line:] http://www.heritagegeophysics.com/images/ lokenote.pdf [access: 24.03.2018].

Loke M.H., 2000. Topographic modelling in resistivity imaging inversion. [in:] Extended Abstracts Book, EAGE 62nd Conference and Technical Exhibition: Glasgow, Scotland, 29 May - 2 June 2000, European Association of Geoscientists \& Engineers, D-2.

Loke M.H., Chambers J.E., Rucker D.F., Kuras O. \& Wilkinson P.B., 2013. Recent developments in the direct-current geoelectrical imaging method. Journal of Applied Geophysics, 95, 135-156.

Pazdirek O. \& Blaha V., 1996. Examples of resistivity imaging using ME-100 resistivity field acquisition system. [in:] Extended Abstracts Book 58th EAGE Conference and Technical Exhibition, Amsterdam, The Netherlands, 3-7 June 1996, European Association of Geoscienists \& Engineers. DOI: 10.3997/2214-4609.201408817. 
Rønning J.S., Dalsegg E., Elvebakk H. \& Storrø G., 2003. Characterization of fracture zones in bedrock using $2 \mathrm{D}$ resistivity. [in:] 9th European Meeting of Environmental and Engineering Geophysics, August 31 - September 4, Prague, Czech Republic, Czech Association of the Applied Geophysicists, Czech Republic, P-005. DOI: 10.3997/2214-4609.201414578.

Roy A. \& Apparao A., 1971. Depth of investigation in direct current methods. Geophysics, 36, 943-959.

Sasaki Y., 1989. Two-dimensional joint inversion of magnetotelluric and dipole-dipole resistivity data. Geophysics, $54,254-262$.
Seaton W.J. \& Burbey T.J., 2002. Evaluation of two-dimensional resistivity methods in a fractured crystalline-rock terrain. Journal of Applied Geophysics, 51, 21-41.

Stummer P., Maurer H. \& Green A.G., 2004. Experimental design: electrical resistivity data sets that provide optimum subsurface information. Geophysics, 69, 1, 120-139.

White R.M.S., Collins S. \& Loke M.H., 2003. Resistivity and IP arrays, optimised for data collection and inversion. Exploration Geophysics, 34, 229-232. 